\title{
Go ahead, make my DNA
}

The reliable and cost-effective synthesis of custom gene sequences is crucial for applications in synthetic biology and biotechnology. Although DNA microarrays can be an economical source of short-oligonucleotide pools for gene synthesis, these complex mixtures must be amplified and correctly assembled. A recent study describes a method whereby 30 genes (or pools of gene variants) can be synthesized on a single chip. One round of synthesis and selection can successfully identify those gene variants with desired properties, such as optimized expression levels.

In their method, Quan et al. performed isothermal nicking and strand-displacement amplification reactions to simultaneously amplify

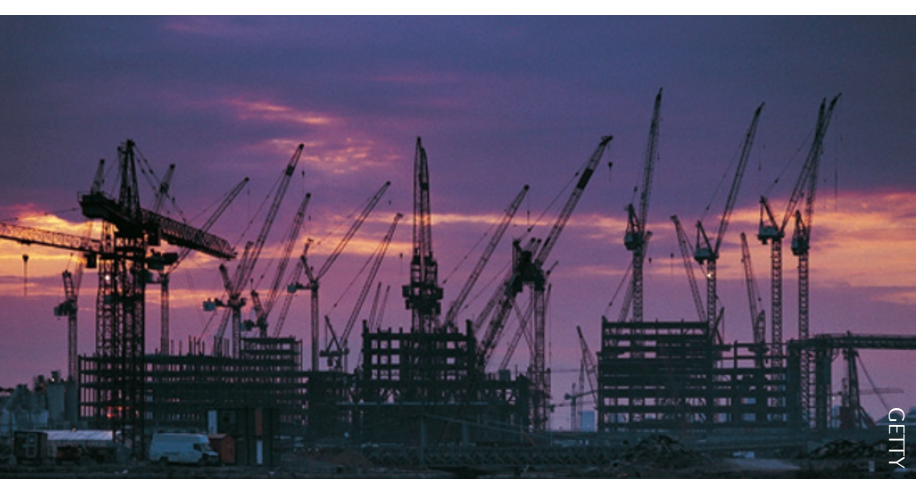

and release 60-mer oligonucleotides, followed by polymerase cycling assembly to build the overlapping products into genes of $0.5-1 \mathrm{~kb}$. For ease, both of these steps were performed on-chip and in the same reaction mixture; spurious oligonucleotide hybridizations were minimized by subdividing the chip into separate wells for each gene. The products were then further amplified by off-chip PCR.

The authors also developed a quality-control procedure in which the subpopulation of erroneous synthetic genes (which form mismatched duplexes after a denaturationreannealing cycle) can be cleaved by mismatch-specific endonucleases. However, a limitation of this procedure is that it is only applicable to the synthesis of single genes, rather than a pooled library of closely related gene variants, owing to the mismatch potential of variants.

The gene-synthesis method was then applied to optimize transgene expression, which requires appropriate codon usage in addition to strong promoter sequences. The authors generated a library of synonymous 'codon variants' of the lacZalpha gene fragment and expressed them in Escherichia coli cells. Clones were selected for maximal lacZalpha expression based on their blue colour intensity when grown on $\mathrm{X}$-gal-containing agar. From these clones, lacZ-alpha sequences were identified that greatly enhanced lacZ-alpha expression relative to wild-type sequences.

In a similar but more valuable application, the authors generated and tested codon variant libraries of 74 Drosophila melanogaster transcription factors. Highly expressed variants were required for antibody generation and these were successfully identified based on the fluorescence intensity of GFP fusion proteins.

It will be interesting to learn whether these optimal gene sequences were functionally selected based on properties such as mRNA structure and stability (in addition to codon usage) and how the method is best extended to the synthesis of longer genes.

Darren J. Burgess

ORIGINAL RESEARCH PAPER Quan, J. et al. Parallel on-chip gene synthesis and application to optimization of protein expression. Nature Biotech. 24 Apr 2011 (doi:10.1038/nbt.1847) 